\title{
A stem cell medium containing neural stimulating factor induces a pancreatic cancer stem-like cell-enriched population
}

\author{
YUSAKU WATANABE ${ }^{1}$, KIYOSHI YOSHIMURA ${ }^{1}$, KOICHI YOSHIKAWA ${ }^{2}$, RYOICHI TSUNEDOMI ${ }^{1}$, \\ YOSHITARO SHINDO ${ }^{1}$, SOU MATSUKUMA ${ }^{1}$, NORIKO MAEDA ${ }^{1}$, SHINSUKE KANEKIYO ${ }^{1}$, \\ NOBUAKI SUZUKI ${ }^{1}$, ATSUO KURAMASU ${ }^{3}$, KOUHEI SONODA ${ }^{4}$, KOJI TAMADA $^{5}$, \\ SEI KOBAYASHI ${ }^{6}$, HIDEYUKI SAYA ${ }^{7}$, SHOICHI HAZAMA ${ }^{1}$ and MASAAKI OKA ${ }^{1}$
}

\begin{abstract}
Departments of ${ }^{1}$ Digestive Surgery and Surgical Oncology, ${ }^{2}$ Neurosurgery, ${ }^{3}$ Molecular Pharmacology, ${ }^{4}$ Ophthalmology,
${ }^{5}$ Immunology and Cell Signaling Analysis, ${ }^{6}$ Molecular Physiology and Medical Bioregulation, Yamaguchi University

School of Medicine, Ube, Yamaguchi 755-8505; ${ }^{7}$ Division of Gene Regulation, Institute for Advanced Medical Research, Graduate School of Medicine, Keio University, Shinjuku-ku, Tokyo 160-8582, Japan
\end{abstract}

Received June 11, 2014; Accepted August 1, 2014

DOI: $10.3892 /$ ijo.2014.2603

\begin{abstract}
Cancer stem cells (CSCs) have been studied for their self-renewal capacity and pluripotency, as well as their resistance to anticancer therapy and their ability to metastasize to distant organs. CSCs are difficult to study because their population is quite low in tumor specimens. To overcome this problem, we established a culture method to induce a pancreatic cancer stem-like cell (P-CSLC)-enriched population from human pancreatic cancer cell lines. Human pancreatic cancer cell lines established at our department were cultured in CSC-inducing media containing epidermal growth factor (EGF), basic fibroblast growth factor (bFGF), leukemia inhibitory factor (LIF), neural cell survivor factor-1 (NSF-1), and N-acetylcysteine. Sphere cells were obtained and then transferred to a laminin-coated dish and cultured for approximately two months. The surface markers, gene expression, aldehyde dehydrogenase (ALDH) activity, cell cycle, and tumorigenicity of these induced cells were examined for their stem cell-like characteristics. The population of these induced cells expanded within a few months. The ratio of CD24high, CD44high, epithelial specific antigen (ESA) high, and CD44variant (CD44v) high cells in the induced cells was greatly enriched. The induced cells stayed in the G0/G1 phase and demonstrated mesenchymal and stemness properties. The induced cells had high tumorigenic potential. Thus, we established a culture method to induce a P-CSLC-
\end{abstract}

Correspondence to: Professor Masaaki Oka, Department of Digestive Surgery and Surgical Oncology, Yamaguchi University School of Medicine, Minami-Kogushi 1-1-1, Ube, Yamaguchi 755-8505, Japan

E-mail: 2geka-1@yamaguchi-u.ac.jp

Key words: cancer stem cells, cell culture method, pancreatic cancer enriched population from human pancreatic cancer cell lines. The CSLC population was enriched approximately 100-fold with this method. Our culture method may contribute to the precise analysis of CSCs and thus support the establishment of CSC-targeting therapy.

\section{Introduction}

CSCs have been studied in terms of their self-renewal capability and pluripotency, as well as their resistance to anticancer therapy and ability to metastasize to distant organs $(1,2)$. Conventional chemotherapies and radiation therapies were initially developed targeting the cancer-cell population. However, these treatments have no efficacy against CSCs, which have been shown to be resistant to standard chemotherapeutic agents (3-5). Pancreatic cancer is the 5th most common cause of cancer death in Japan (Center for Cancer Control and Information Services, National Cancer Center, Japan). The overall 5-year survival rate worldwide is $<10 \%$ (6). The prognosis for pancreatic cancer patients with hepatic metastases is dismal because these patients cannot have radical surgery. Thus, novel and effective treatments against pancreatic CSCs are greatly needed. CSCs can be identified and isolated by different methodologies, including isolation by CSC-specific cell surface marker expression, detection of side population phenotype by Hoechst 33342 exclusion, and assessment of their ability to grow as floating spheres (7-13). However, the population of CSCs in tumor specimens is quite low; therefore, it is difficult to obtain purified CSCs in adequate numbers for effective study. To overcome this problem, we established a culture method to induce a P-CSLC-enriched population from human pancreatic cancer cell lines. In long-term culture, these induced cells maintained their stem-like phenotype as characterized by: i) the ability to survive under harsh conditions created by the media without serum and with EGF, bFGF, LIF, and NSF-1, in which non-stem-like cancer cells are not able to survive; ii) sphere-shaped morphology; and iii) longer survival in laminin-coated dishes. This method is stable and durable 
and will support the establishment of CSC-targeting therapy by consistently providing abundant CSCs.

\section{Materials and methods}

Culture of human pancreatic cancer cell lines. The human cancer cell lines used in the experimental study were pancreatic cancer cell lines YPK2 and YPK5, which were established in our department (14). Cell lines were maintained in DMEM-F12 (Sigma-Aldrich, Tokyo, Japan) containing 10\% heat-inactivated FBS (Life Technologies, Tokyo, Japan) at $37^{\circ} \mathrm{C}$ in $5 \% \mathrm{CO}_{2}$.

Induction and culture of CSLC-enriched population. Cells were initially cultured in serum-free medium which is based on neural stem cell medium. The basal medium for the sphere induction is DMEM-F12 supplemented with $10 \mathrm{mM}$ HEPES (Sigma-Aldrich), 1X antibiotic antimycotic solution (SigmaAldrich), 0.6\% glucose (Sigma-Aldrich), $1 \mathrm{mg} / \mathrm{ml}$ transferrin, $250 \mu \mathrm{g} / \mathrm{ml}$ insulin (Sigma-Aldrich), $0.6 \mathrm{mM}$ putrescine (Sigma-Aldrich), 0.3 $\mu \mathrm{M}$ sodium selenite (Sigma-Aldrich), and $0.2 \mu \mathrm{M}$ progesterone (Sigma-Aldrich). Complete sphere induction medium was prepared by adding $2 \mu \mathrm{g} / \mathrm{ml}$ heparin (Sigma-Aldrich), $20 \mathrm{ng} / \mathrm{ml}$ EGF (Sigma-Aldrich), $20 \mathrm{ng} / \mathrm{ml}$ bFGF (Merck Millipore, Tokyo, Japan), 10 ng/ml LIF (Merck Millipore), 1/50 Vol NSF-1 (Lonza, Tokyo, Japan), and $60 \mu \mathrm{g} / \mathrm{ml} \mathrm{N}$-acetyl-L-cysteine (Sigma-Aldrich). Upon the formation of spheres, the sphere cells (YPK2-Sp and YPK5-Sp) were collected. YPK2-Sp or YPK5-Sp were then transferred to a laminin-coated dish with the sphere culture medium containing $20 \mu \mathrm{l} / \mathrm{ml} \mathrm{B27}$ supplement (Life Technologies), $1 \mathrm{X}$ antibiotic antimycotic solution, $75 \mu \mathrm{g} / \mathrm{ml}$ BSA (SigmaAldrich), $10 \mathrm{ng} / \mathrm{ml}$ EGF, and $10 \mathrm{ng} / \mathrm{ml} \mathrm{bFGF}$. Medium was renewed by a $50 \%$ change every 7 days. Cells became attached and gradually divided and increased in number (YPK2-Lm and YPK5-Lm).

Flow cytometry analysis and sorting. Dissociated cells were counted and transferred to a 5-ml tube, washed twice with PBS containing 2\% heat-inactivated FBS, and resuspended in PBS with $2 \%$ FBS at a concentration of $10^{6}$ cells per $100 \mu 1$. Antibodies at the appropriate dilution were added to the cells, and the mixture was incubated for $20 \mathrm{~min}$ on ice. Then, the sample was washed twice with PBS containing $2 \%$ FBS. The antibodies were anti-CD44 allophycocyanin (APC) (eBioscience, San Diego, CA, USA), anti-CD24 phycoerythrin (PE) (Beckman Coulter, Brea, CA, USA), anti-ESA-FITC (GeneTex, Irvine, CA, USA), and anti-CD44v, which was kindly provided by Dr Hideyuki Saya (Keio University, Tokyo, Japan). Flow cytometry analysis was performed by using a MACSQuant analyzer (Miltenyi Biotec, Gladbach, Germany), and results were analyzed with FlowJo software (TreeStar, OR, USA). CD24high/CD44high cells were then isolated and sorted from YPK-Lm by FACSAria III (BD Immunocytometry Systems, Franklin Lakes, NJ, USA). The sorted CD24high/CD44high cells were referred to as YPK2-SortLm and YPK5-SortLm.

Analysis of ALDHactivity. To assess the cellular ALDHactivity, the Aldefluor assay kit (StemCell Technologies, Vancouver, BC, Canada) was used according to the manufacturer's guidelines. Briefly, cells were harvested, placed in Aldefluor assay buffer $\left(1 \times 10^{6} / \mathrm{ml}\right)$, and incubated with Aldefluor substrate for $45 \mathrm{~min}$ at $37^{\circ} \mathrm{C}$ to allow substrate conversion. As a negative control for all experiments, an aliquot of Aldefluor-stained cells was immediately quenched with $1.5-\mathrm{mM}$ diethylaminobenzaldehyde (DEAB), a specific ALDH inhibitor. Cells were analysed by using the green fluorescence channel (FL1) on a MACSQuant analyzer, and results were analyzed with FlowJo software. Cells that fell within the closed area were considered to represent subpopulations of cells with enhanced ALDH activity as compared with the rest of the cell population.

Cell cycle phase distribution analysis. We performed the cell cycle analysis according to company recommendations (BD Bioscience, Franklin Lakes, NJ, USA). Briefly, cells were trypsinized and centrifuged at $1500 \mathrm{rpm}$ for $5 \mathrm{~min}$, washed twice with PBS, and then fixed with $70 \%$ cold ethanol. Fixed cells were stained by using PI/RNase Staining Buffer (BD Bioscience) and incubated for $15 \mathrm{~min}$ at room temperature before analysis. Analysis was performed with the MACSQuant analyzer, and results were analyzed with FlowJo software.

Xenograft model. $\mathrm{Rag}^{-/-}$IL-2 common gamma chain ${ }^{-/-}$mice were purchased from the Jackson Laboratory (Bar Harbor, ME, USA) and bred and maintained in a HEPA-filtered environment with autoclave-sterilized cages, food, and bedding. All animal studies were conducted in accordance with the Institutional Animal Care and Use Committee of Yamaguchi University and conformed to the Guide for the Care and Use of Laboratory Animals published by the US National Institutes of Health. Mice were inoculated with $10^{3}$ or $10^{4}$ cells in each experiment. All mice were inoculated subcutaneously in the left lower abdominal quadrant with a 27 -gauge needle.

Semi-quantitative real-time $R T-P C R$. The expression levels of stemness genes (KIT, ALDH1A1, NANOG) and epithelialmesenchymal transition (EMT)-related genes (CDH1, CDH2, VIM, FN1, SNAI1, SNAI2, ZEB1, ZEB2) were examined by RT-PCR. Semi-quantitative real-time RT-PCR was performed as described previously with minor modifications $(15,16)$. RNAs were extracted from cells by using TRIzol reagent (Life Technologies). Reverse transcription was performed with the PrimeScriptRT reagentkit(Takara Bio, Shiga,Japan). Real-time PCR amplification was performed by using LightCycler 480 Probe Master (Roche Diagnostics, Tokyo, Japan) and Universal ProbeLibrary probes (Roche Diagnostics) in a LightCycler System Version 3 (Roche Diagnostics). Primers and probes are listed in Table I. Amplification was performed according to a 2-step cycle procedure consisting of 45 cycles of denaturation at $95^{\circ} \mathrm{C}$ for $10 \mathrm{sec}$ and annealing/elongation at $60^{\circ} \mathrm{C}$ for $30 \mathrm{sec}$. We measured mRNA levels semi-quantitatively by the $\Delta / \Delta$ threshold cycle $(\mathrm{Ct})$ method. Both the GAPDH and $\beta$-actin (ACTB) genes were used as reference genes. The values are expressed as relative to the parental cells.

Measurements of cytokine and chemokine levels. Frozen aliquots of YPK2 and YPK5 were thawed and cultured for 2 weeks prior to harvesting culture supernatant from sub-confluent cultures (Sup-YPK2 and Sup-YPK5). YPK2-Lm and YPK5-Lm supernatant was harvested when cells were sub-confluent 1 month after transfer to laminin-coated dishes 
Table I. Primers and probes.

\begin{tabular}{|c|c|c|c|}
\hline Symbol & Name & UPL probe no. & Sequence $\left(5^{\prime}-3^{\prime}\right)$ \\
\hline KIT (C-Kit, CD117) & $\begin{array}{l}\text { KIT-S } \\
\text { KIT-AS }\end{array}$ & 71 & $\begin{array}{l}\text { ctttcctcgcctccaagaat } \\
\text { gtgatccgaccatgagtaagg }\end{array}$ \\
\hline ALDH1A1 & $\begin{array}{l}\text { ALDH1A1-S } \\
\text { ALDH1A1-AS }\end{array}$ & 14 & $\begin{array}{l}\text { tttggtggattcaagatgtctg } \\
\text { cactgtgactgttttgacctctg }\end{array}$ \\
\hline NANOG & $\begin{array}{l}\text { NANOG-S } \\
\text { NANOG-AS }\end{array}$ & 31 & $\begin{array}{l}\text { agatgcctcacacggagact } \\
\text { tttgcgacactcttctctgc }\end{array}$ \\
\hline CDH1 (E-cadherin) & $\begin{array}{l}\text { CDH1-S } \\
\text { CDH1-AS }\end{array}$ & 35 & $\begin{array}{l}\text { cccgggacaacgtttattac } \\
\text { gctggctcaagtcaaagtcc }\end{array}$ \\
\hline CDH2 (N-cadherin) & $\begin{array}{l}\mathrm{CDH} 2-\mathrm{S} \\
\mathrm{CDH} 2-\mathrm{AS}\end{array}$ & 80 & $\begin{array}{l}\text { agtatccggtccgatctgc } \\
\text { ctgtggggtcattgtcagc }\end{array}$ \\
\hline VIM (vimentin) & $\begin{array}{l}\text { VIM-S } \\
\text { VIM-AS }\end{array}$ & 13 & $\begin{array}{l}\text { tacaggaagctgctggaagg } \\
\text { accagagggagtgaatccag }\end{array}$ \\
\hline FN1 & $\begin{array}{l}\text { FN1-S } \\
\text { FN1-AS }\end{array}$ & 60 & $\begin{array}{l}\text { aagagcgagcccetgatt } \\
\text { atgaagattggggtgtggaa }\end{array}$ \\
\hline SNAI1 & $\begin{array}{l}\text { SNAI1-S } \\
\text { SNAI1-AS }\end{array}$ & 10 & $\begin{array}{l}\text { catgtccggacccacact } \\
\text { tggcactggtacttcttgaca }\end{array}$ \\
\hline SNAI2 (SLUG) & $\begin{array}{l}\text { SNAI2-S } \\
\text { SNAI2-AS }\end{array}$ & 7 & $\begin{array}{l}\text { tggttgcttcaaggacacat } \\
\text { gttgcagtgagggcaagaa }\end{array}$ \\
\hline ZEB1 & $\begin{array}{l}\text { ZEB1-S } \\
\text { ZEB1-AS }\end{array}$ & 36 & $\begin{array}{l}\text { cctaaaagagcacttaagaattcacag } \\
\text { catttcttactgcttatgtgtgagc }\end{array}$ \\
\hline ZEB2 (SIP1) & $\begin{array}{l}\text { ZEB2-S } \\
\text { ZEB2-AS }\end{array}$ & 68 & $\begin{array}{l}\text { aagccagggacagatcagc } \\
\text { ccacactctgtgcatttgaact }\end{array}$ \\
\hline
\end{tabular}

in the sphere culture medium (Sup-Lm2 and Sup-Lm5). The Bioplex assay (Bio-Rad, Marne la Coquette, France) was performed according to the manufacturer's instructions to evaluate the levels of cytokines and chemokines in the supernatant. Samples were analyzed in triplicate. Experimental data were analyzed by using five-parametric curve fitting. We measured the protein level of the following 28 cytokines and chemokines: TGF- $\beta$, IL-1b, IL-1ra, IL-2, IL-4, IL-5, IL-6, IL-7, IL-8, IL-9, IL-10, IL-12, IL-13, IL-17, eotaxin, bFGF, G-CSF, GM-CSF, interferon (IFN) $-\gamma$, immune protein (IP)-10, monocyte chemotactic protein (MCP)-1, macrophage inflammatory proteins (MIP)-1 $\alpha$, MIP-1 $\beta$, platelet-derived growth factor (PDGF)-BB, regulated on activation, normal T-cell expressed and secreted (RANTES), tumor necrosis factor (TNF)- $\alpha$, and vascular endothelial growth factor (VEGF).

Statistical analysis. The results are presented as means \pm SD. Statistical differences were determined using the MannWhitney U tests. P-values of $<0.05$ were considered significant.

\section{Results}

Induction and culture of CSLC-enriched population. When YPK2 or YPK5 were initially cultured in the CSC-inducing media, cells began to attach on the plate, and a portion of cells formed spheres in suspension culture within a few hours (YPK2-Sp and YPK5-Sp) (Fig. 1A and D). These spheres grew to become larger sphere clusters within a week. YPK2-Sp or YPK5-Sp were harvested on day 7 and transferred to laminincoated dishes. Cells began to attach to the dishes within a few hours; then, they gradually divided and the number of spheres and attached cells increased for 2 months (YPK2-Lm and YPK5-Lm, Fig. 1B, C, E and F). The surviving cells displayed both attached and cluster-formatted morphology. When these cells were grown in culture for $>3$ months, they became apoptotic without proliferation. Fig. $1 \mathrm{G}$ and $\mathrm{H}$ show YPK2 and YPK5 cultured in DMEM containing 10\% FBS. These cells were attached and proliferated quickly.

Cell surface markers. In general, a CD $44^{+} / \mathrm{CD} 24^{+} / \mathrm{ESA}^{+}$ phenotype has stem-cell properties in pancreatic cancer cells (7). In the present study, the ratio of the expression of CD24high/CD44high in YPK2 (Fig. 2A) and YPK5 (Fig. 2D) was $\sim 0.1 \%$, while the ratios (mean $\pm \mathrm{SD}$ ) in YPK2-Lm (Fig. 2B) and YPK5-Lm (Fig. 2E) were significantly increased to $7.5 \pm 2.6 \%(\mathrm{P}=0.0211)$ and $11.1 \pm 2.8 \%(\mathrm{P}=0.0211)$, respectively. Expression of ESA in YPK2-SortLm (23.2\%) and YPK5-SortLm (36.2\%) was higher than that of YPK2 $(0.1 \%)$ and YPK5 (0.1\%) (Fig. 2C and F). Recently, some studies have focused on the role of CD44v in CSCs $(17,18)$. We also focused 

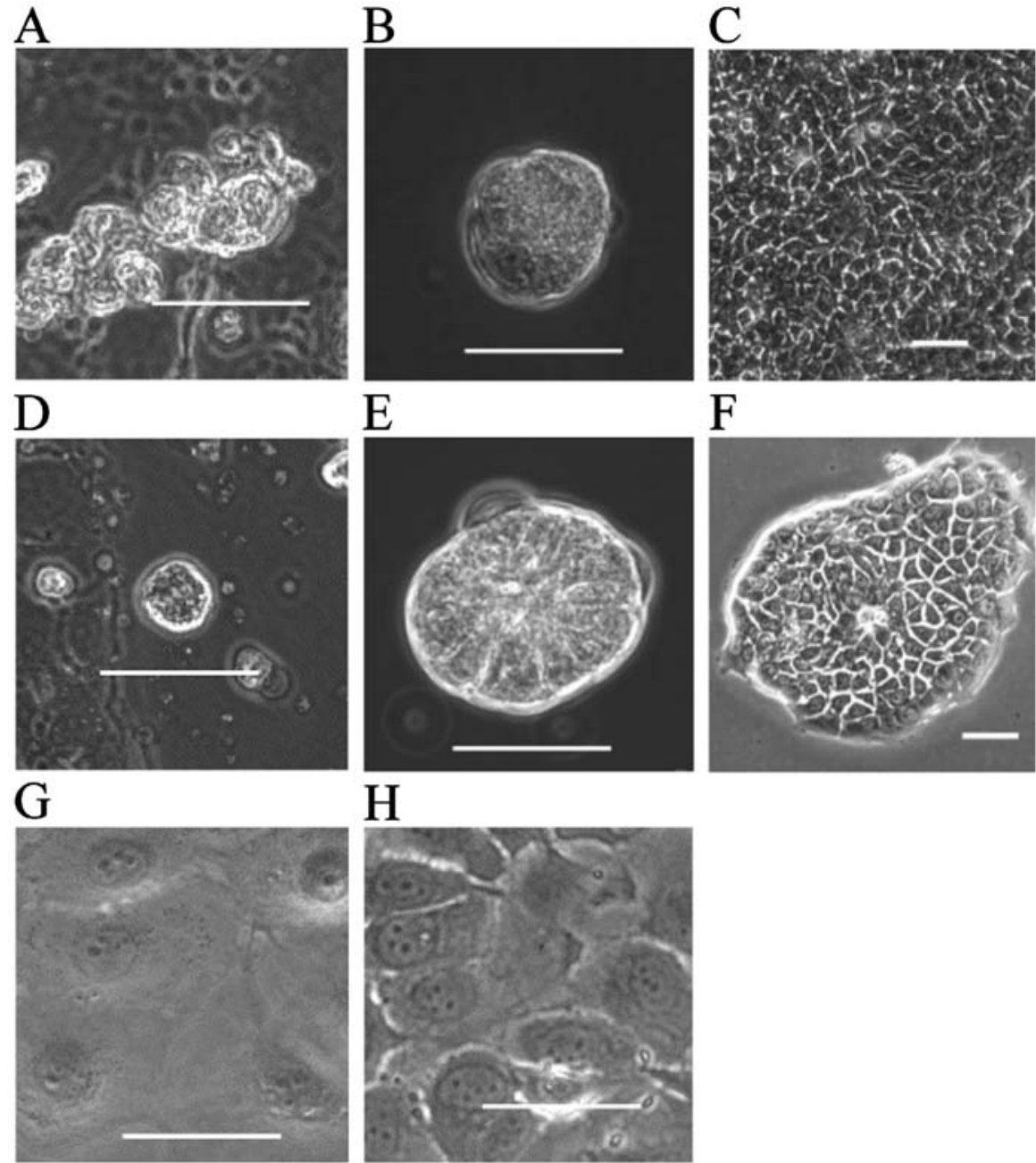

Figure 1. Sphere formation and induction of CSLC-enriched population. When YPK2 and YPK5 were initially cultured in the CSC-inducing media, a portion of cells formed spheres in suspension culture in a few hours [(A) YPK2; (D) YPK5]. These sphere cells were harvested on day 7 and transferred to laminin-coated dishes. (B) One week after YPK2-Sp was transferred to laminin-coated dishes. (C) Two months after YPK2-Sp was transferred to laminin-coated dishes. (E) One week after YPK5-Sp was transferred to laminin-coated dishes. (F) Two months after YPK5-Sp was transferred to laminin-coated dishes. YPK2 and YPK5 cultured in DMEM containing 10\% FBS maintained the same morphology as parental cells [(G) YPK2; (H) YPK5]. Scale bars, $50 \mu \mathrm{m}$.

on the expression of CD44v. Fig. 2G shows that YPK2-SortLm expressed a higher ratio of CD44v than YPK2-Lm and YPK2. The ratio of CD44v in YPK2 was only $0.2 \%$; however, this ratio in YPK2-Lm and YPK2-SortLm was 16.7 and 99.8\%, respectively. Expression of CD44v in YPK5-SortLm was also high compared to YPK5 (Fig. 2H). When NSF-1 or LIF was omitted from CSC-inducing media, CD24low/CD44low cells were dominant; these cells were also dominant in parent cancer cells (Fig. 2I and J).

ALDH activity and cell cycle analysis. A functional mechanism for chemo-resistance has been associated with ALDH activity (19). In the present study, YPK2 and YPK5 expressed high levels of ALDH activity (YPK2, 68.5\%; YPK5, 54.5\%), however, YPK2-Lm and YPK5-Lm expressed much higher levels of ALDH activity (YPK2-Lm, 93.4\%; YPK5-Lm,92.0\%) than parental cells (Fig. 3).

Stem cell quiescence is also highly relevant for chemotherapy against cancer, as it is retained and contributes to relapse following discontinuation of therapy (20). Many CSCs are non-cycling G0 cells and would not be susceptible to cell cycle-specific chemotherapy agents. Many of YPK2-Lm and YPK5-Lm are relatively quiescent compared to the YPK2 and YPK5, however, these were not statistically significant (Fig. 4).

Tumorigenicity. YPK2-SortLm cells gave rise to new tumors in 3 of 3 mice, $\geq 10^{3}$ cells were injected. In contrast, no tumors formed when $10^{3}$ YPK2 cells were injected, which demonstrates the much higher tumorigenicity of YPK2-SortLm cells.

mRNA expression of stem-cell and mesenchymal markers. The theory of the relationship between EMT and CSCs has been supported recently by the fact that cancer cells with migratory and invasive capabilities associated with metastatic competence are caused through EMT (21-23). Recent studies have established a crucial link between passage through EMT and the acquisition of the molecular and functional properties of stem cells $(24,25)$. We therefore confirmed whether YPK-Lm have EMT properties (Fig. 5). RT-PCR resulted in significantly higher expression levels of stemness genes such 
A

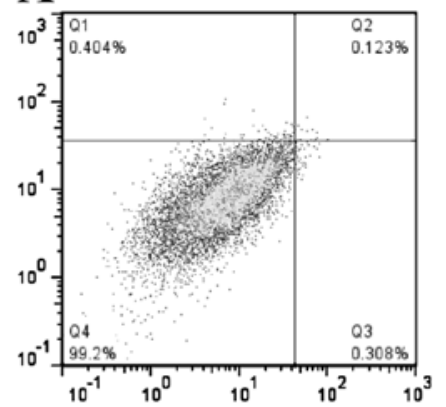

D

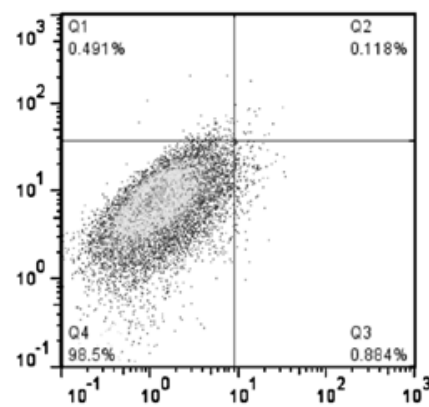

G

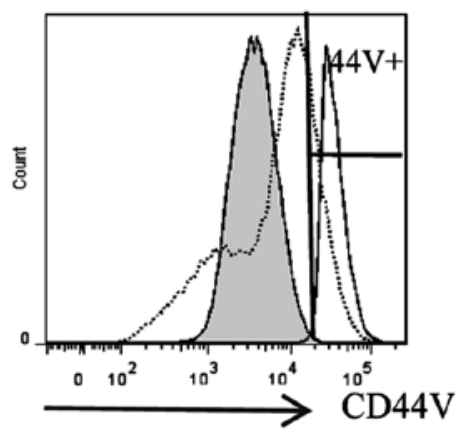

B

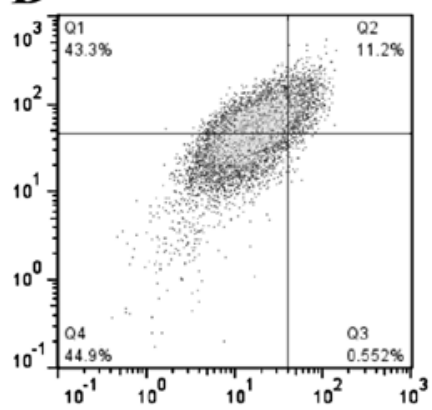

E

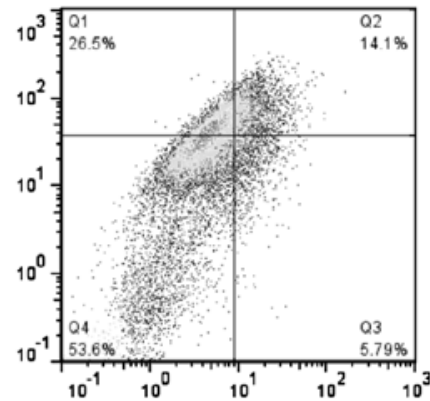

C
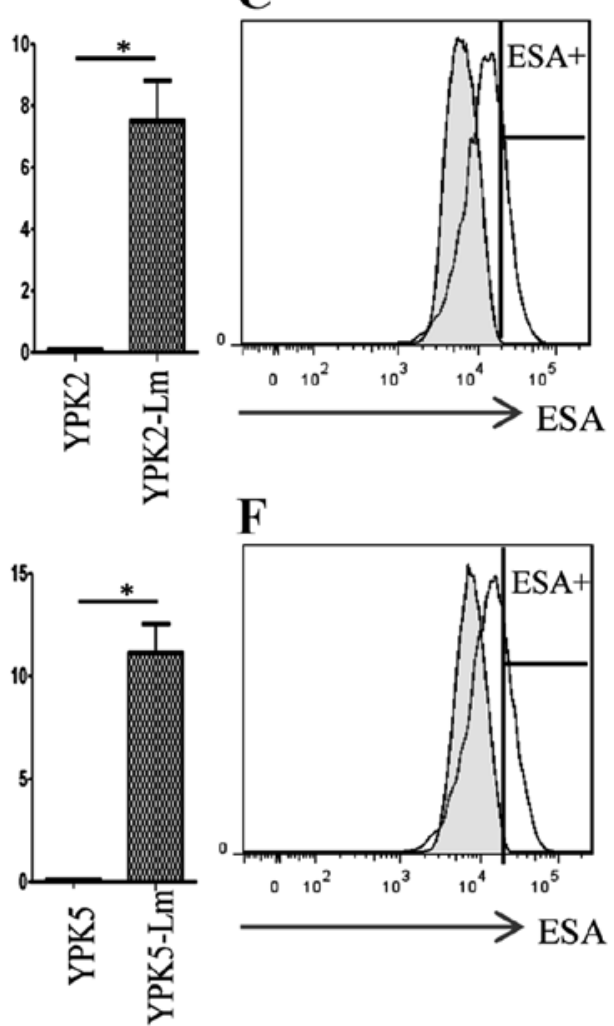

F

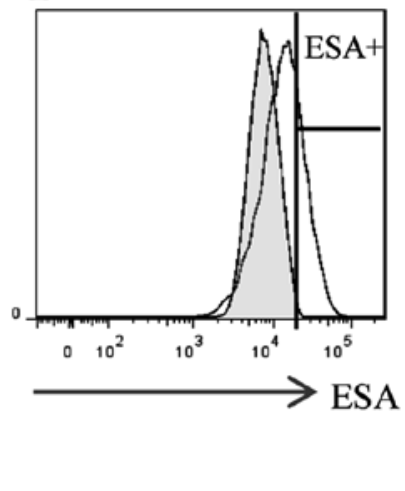

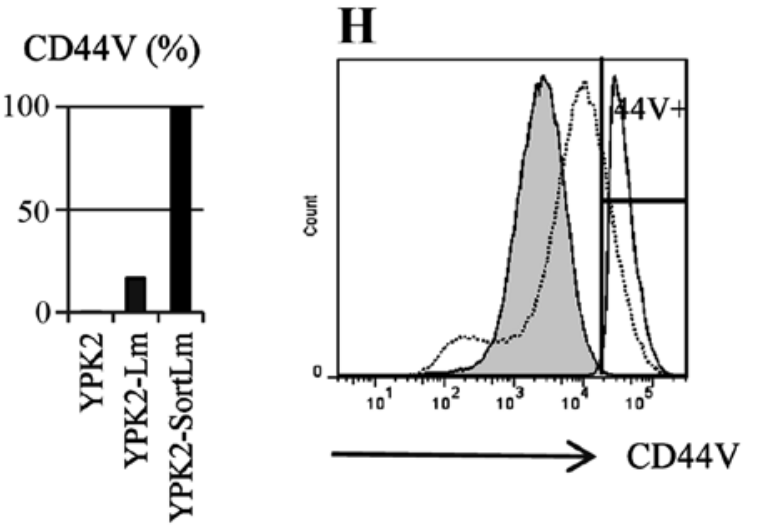

CD44V (\%)

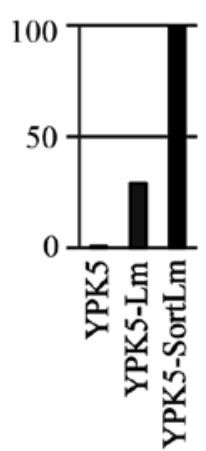

I

CD44

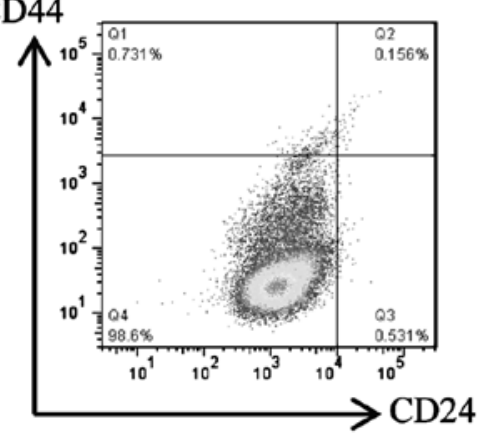

J

CD44

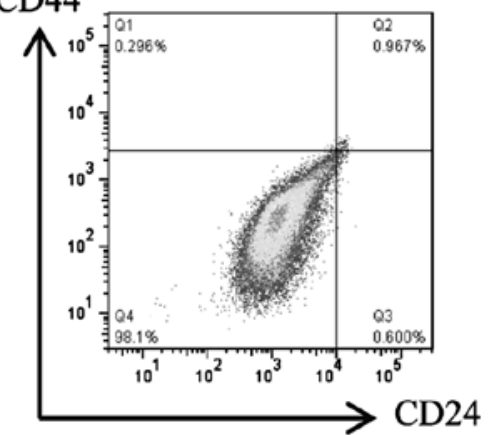

Figure 2. Expression of CD24, CD44, CD44v and ESA. The ratio of the expression of CD24high/CD44high in YPK2 and YPK5 was 0.1\% [(A) YPK2; (D) YPK5]. The ratios of the expression of CD24high/CD44high in YPK2-Lm and YPK5-Lm were 7.5 \pm 2.6 and 11.1 $2.8 \%$, respectively [(B) YPK2-Lm; (E) YPK5-Lm]. Expression of ESA in YPK2-SortLm [(C) black line, 23.2\%] and YPK5-SortLm [(F) black line, 36.2\%] was higher than that of YPK2 [(C) tinted line, $0.1 \%$ ] and YPK5 [(F) tinted line, $0.1 \%]$. (G) The ratio of CD44v in YPK2 (tinted line) was only 0.2\%, but the ratios in YPK2-Lm (dotted line) and YPK2-SortLm (black line) were increased to 16.7 and 99.8\%, respectively. $(\mathrm{H})$ The ratio of CD44v in YPK5 (tinted line) was only $0.2 \%$, however these ratios of CD44v in YPK5-Lm (dotted line) and YPK5-SortLm (black line) were 16.7 and 99.8\%, respectively. (I) YPK 2-Lm was cultured in the CSC-inducing media without NSF-1. (J) YPK 2-Lm was cultured in the CSC-inducing media without LIF. When NSF-1 or LIF were omitted from the CSC-inducing media, cells expressed cancer cell-like patterns of surface markers such as CD24low/CD44low. Incidences in (B) and (E) were evaluated by Mann-Whitney U tests; ${ }^{*} \mathrm{P}<0.05$. 

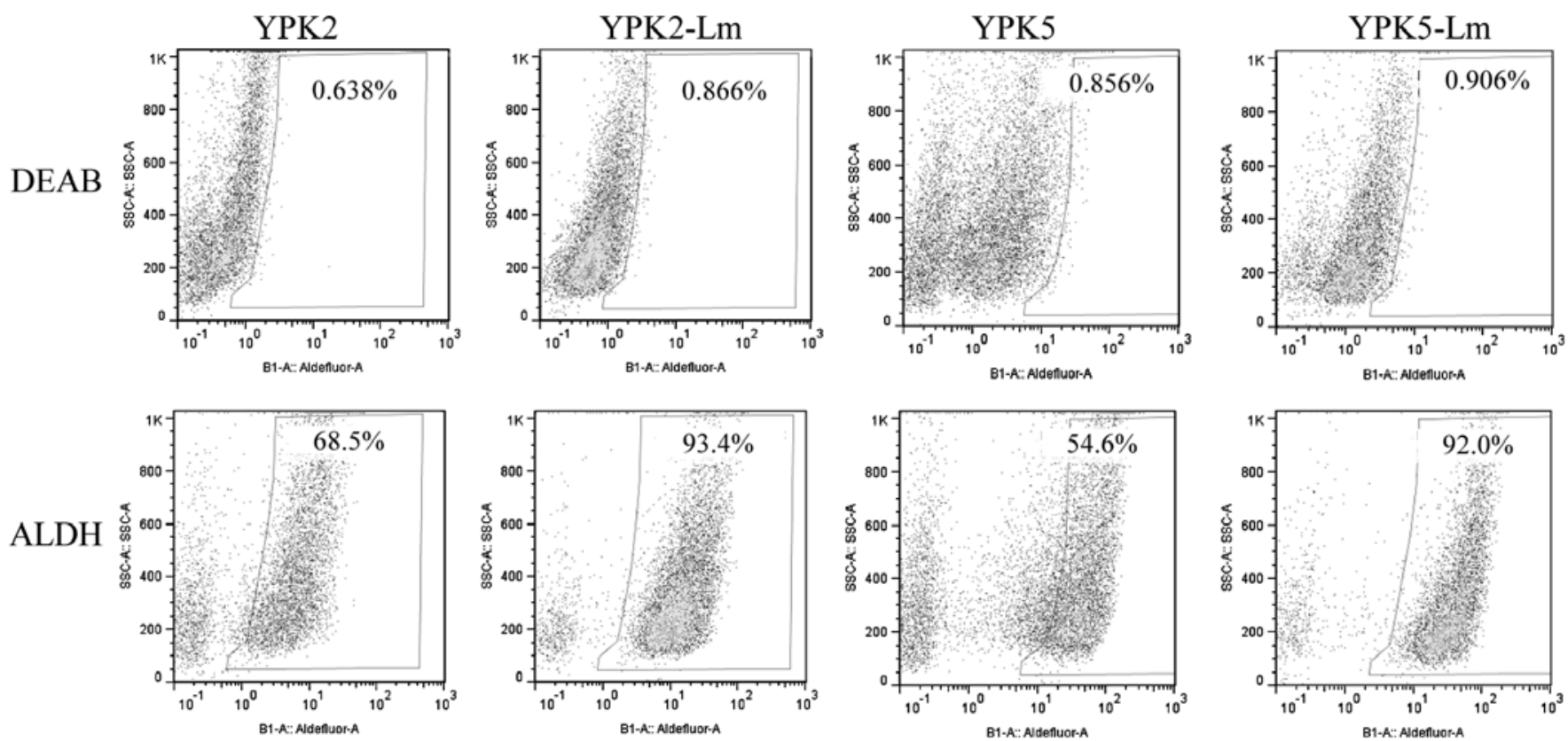

Figure 3. ALDH activity. The upper panels show representative dot plots of cells treated with the ALDH-specific inhibitor DEAB (negative controls). The lower panels show representative dot plots of ALDH activity. YPK2 and YPK5 expressed high levels of ALDH activity (YPK2, 68.5\%; YPK5, 54.6\%), however, YPK2-Lm and YPK5-Lm expressed much higher levels of ALDH activity (YPK2-Lm, 93.4\%; YPK5-Lm, 92.0\%) than parental cells.
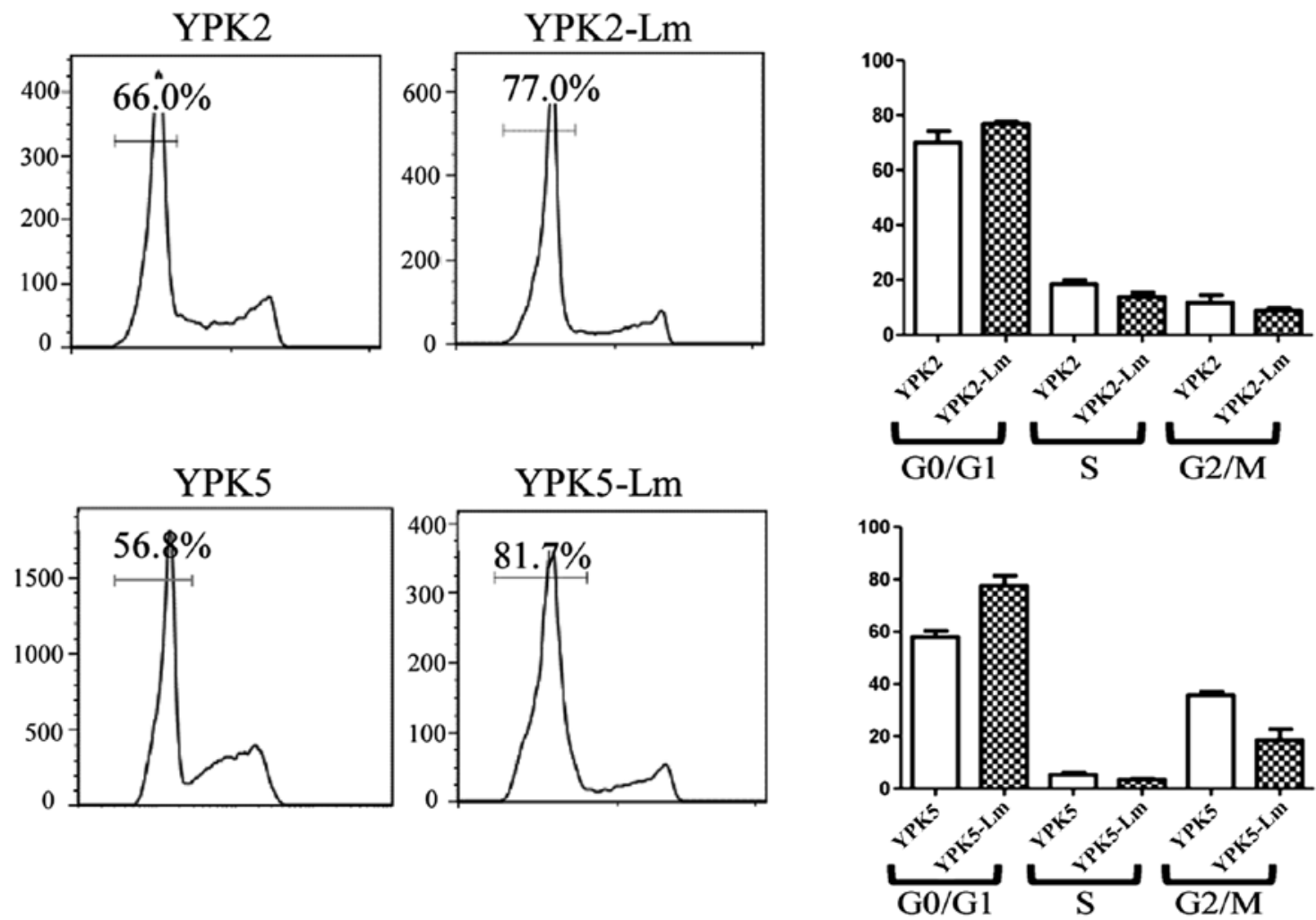

Figure 4. Cell cycle. Many of the YPK2-Lm and YPK5-Lm are relatively quiescent compared to the YPK2 and YPK5, however, these were not statistically significant. Evaluated by the Mann-Whitney U tests.

as KIT and ALDH1A1 in both YPK2-Lm $(\mathrm{P}=0.0095$ and 0.0022 , respectively) and YPK5-Lm $(\mathrm{P}=0.0022$ and 0.0049 , respectively) (Fig. 5A). The expression level of NANOG was significantly high in only YPK2-Lm (YPK2-Lm; P=0.005,
YPK-5Lm; P=0.9361). The expression levels of mesenchymal genes such as CDH2, VIM, SNAI1, SNAI2, ZEB1, ZEB2, and FN1 were significantly higher in YPK2-Lm than in YPK2 $(\mathrm{P}=0.0022$, respectively) (Fig. 5C). In YPK5-Lm, the expres- 


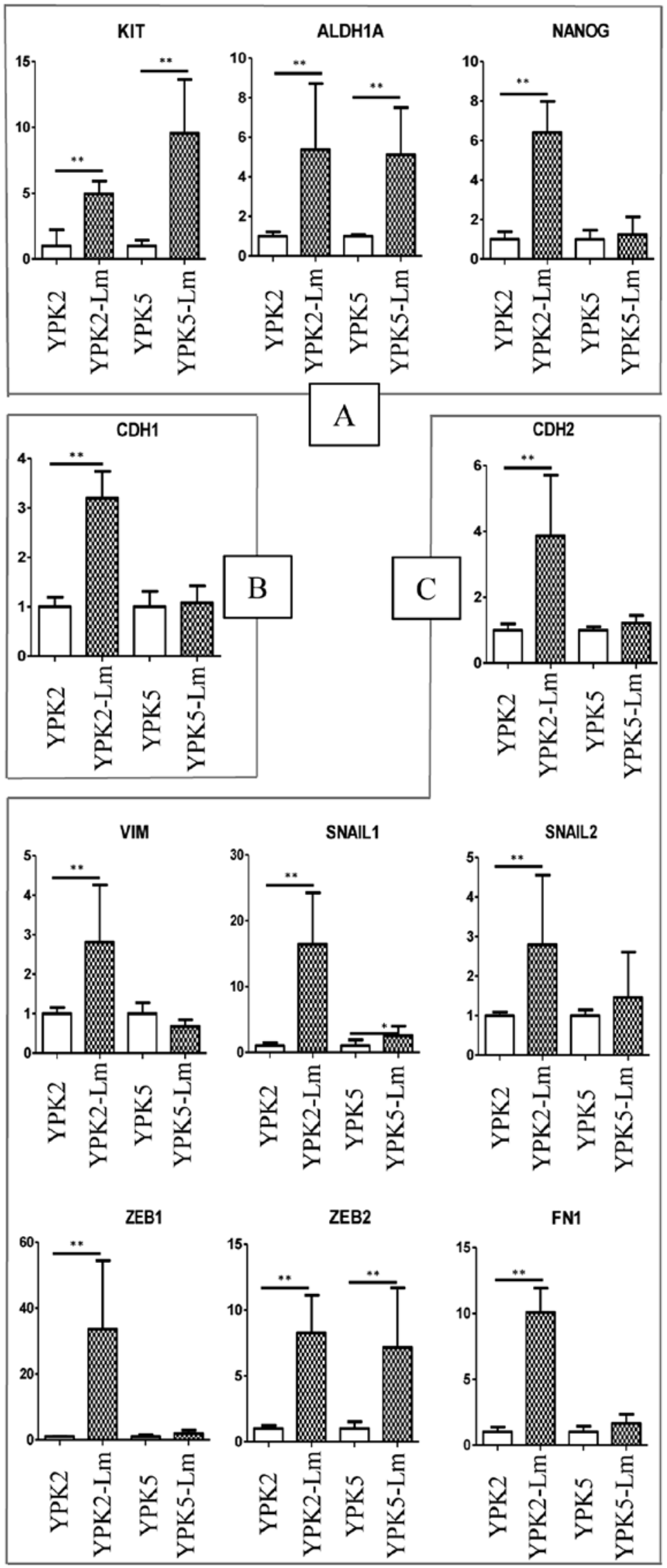

Figure 5. mRNA expression of stemness and EMT-related markers. (A) The expression levels of stemness genes. RT-PCR resulted in significantly higher expression levels of KIT and ALDH1A1 in both YPK2-Lm ( $\mathrm{P}=0.0095$ and 0.0022 , respectively) and YPK5-Lm ( $\mathrm{P}=0.0022$ and 0.0049 , respectively). (B) The expression levels of epithelial genes. The expression level of CDH1 was significantly higher in YPK2-Lm than YPK2, but was not statistically significant between YPK5 and YPK5-Lm. (C) The expression levels of mesenchymal genes. The expression levels of CDH2, VIM, SNAI1, SNAI2, ZEB1, ZEB2, and FN1 were significantly higher in YPK2-Lm than in YPK2 ( $\mathrm{P}=0.0022$, respectively). In YPK5-Lm, the expression levels of SNAI1 and ZEB2 were significantly higher than in YPK5 $\left(\mathrm{P}=0.026\right.$ and 0.0087 , respectively). Evaluated by the Mann-Whitney $\mathrm{U}$ tests; ${ }^{*} \mathrm{P}<0.05 ;{ }^{* *} \mathrm{P}<0.01$. 


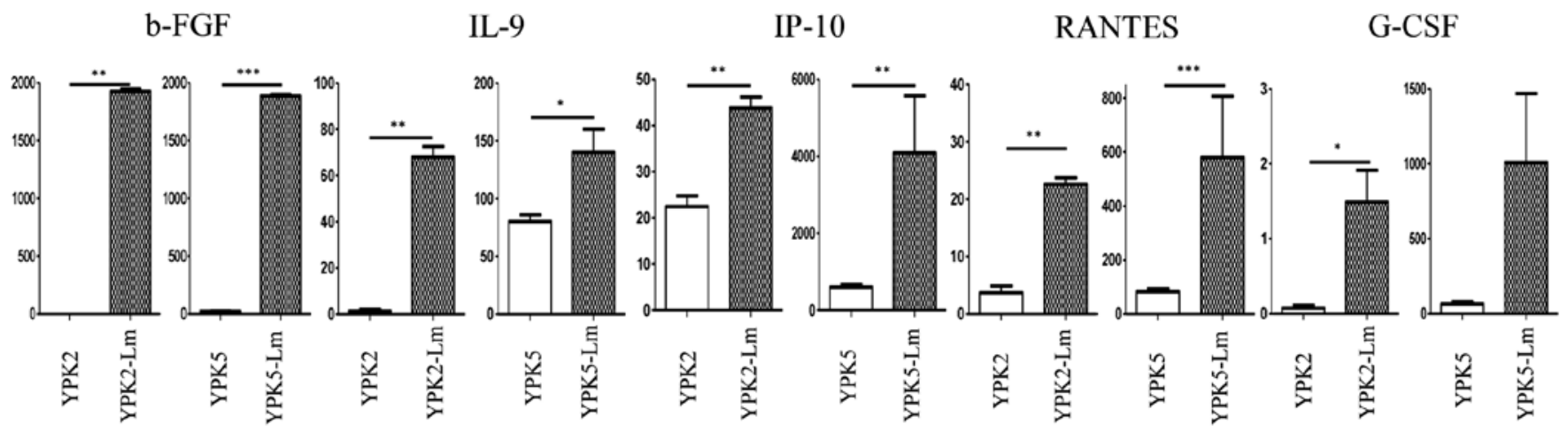

TGF- $\beta 1$

TGF- $\beta 3$

IL-5

IL-12

PDGF-BB

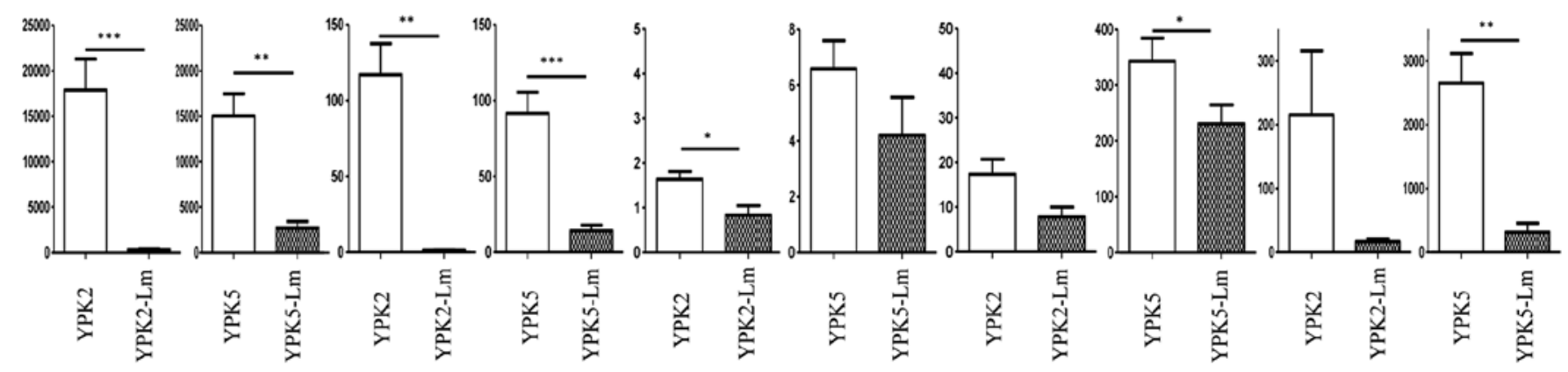

Figure 6. Cytokine assays of culture media. The levels of b-FGF, IL-9, IP-10, and RANTES were significantly detected as higher concentrations in the Sup-Lm 2 and Sup-Lm5 compared to the Sup-YPK2 and Sup-YPK5 (P<0.05). The levels of G-CSF was also significantly detected as higher concentrations in the Sup-Lm 2 compared to the Sup-YPK2 (P=0.02), and also higher trend in the Sup-Lm5 than in the Sup-YPK5 (P=0.06). The levels of TGF- $\beta 1$ and TGF- $\beta 3$ were detected as higher concentrations in the Sup-YPK2 and Sup-YPK5 compared to the Sup-Lm2 and Sup-Lm5 (P<0.01). The level of IL-5 was significantly detected as higher concentrations in the Sup-YPK2 compared to the Sup-Lm2 (P=0.015), and also higher trend in the Sup-YPK5 than in the Sup-Lm5 $(\mathrm{P}=0.07)$. The levels of IL-12 and PDGF-BB were also significantly detected as higher concentrations in the Sup-YPK5 compared to the Sup-Lm5 ( $\mathrm{P}=0.04$ and 0.0027), although these were not statistically significant between the Sup-YPK2 and Sup-Lm2 (P=0.0939 and 0.0926). Evaluated by the Mann-Whitney $\mathrm{U}$ tests; ${ }^{*} \mathrm{P}<0.05 ;{ }^{* *} \mathrm{P}<0.01 ;{ }^{* * *} \mathrm{P}<0.001$.

sion levels of SNAI1 and ZEB2 were significantly higher than in YPK5 ( $\mathrm{P}=0.026$ and 0.0087 , respectively). The expression level of CDH1 was significantly higher in YPK2-Lm than YPK2, but was not statistically significant between YPK5 and YPK5-Lm (Fig. 5B).

Cytokine analysis in the culture media. To question the interaction of microenvironment between cancer and CSLC, we performed multiple cytokine assays with their culture media (Fig. 6). The levels of b-FGF, IL-9, IP-10, and RANTES were significantly detected as higher concentrations in the Sup-Lm2 and Sup-Lm5 compared to the Sup-YPK2 and Sup-YPK5 $(\mathrm{P}<0.05)$. The level of G-CSF was also significantly detected as higher concentrations in the Sup-Lm2 compared to the Sup-YPK2 $(\mathrm{P}=0.02)$, and also higher trend in Sup-Lm5 than in Sup-YPK5 $(\mathrm{P}=0.06)$. The levels of TGF- $\beta 1$ and TGF- $\beta 3$ were detected as higher concentrations in the Sup-YPK2 and Sup-YPK5 compared to the Sup-Lm2 and Sup-Lm5 $(\mathrm{P}<0.01)$. The level of IL-5 was significantly detected as higher concentrations in the Sup-YPK2 compared to the Sup-Lm2 $(\mathrm{P}=0.015)$, and also higher trend in the Sup-YPK5 than in the Sup-Lm5 $(\mathrm{P}=0.07)$. The levels of IL-12 and PDGF-BB were also significantly detected as higher concentrations in the Sup-YPK5 compared to the Sup-Lm5 ( $\mathrm{P}=0.04$ and 0.0027), although these were not statistically significant between the Sup-YPK2 and Sup-Lm2 (P=0.0939 and 0.0926).

\section{Discussion}

We established a novel culture method to induce a P-CSLCenriched population from human pancreatic cancer cell lines. As a first step, human pancreatic cancer cell lines were cultured and induced to form spheres/aggregates within a week. As a second step, these sphere cells were transferred to a laminin-coated dish with the medium, attached and the population of these induced cells expanded within a few months. In the present study, the ratio of CD24high/CD44high cells in YPK-Lm was enriched (Fig. 2B and E). Almost all of YPK-Lm expressed CD44v and also expressed high levels of ALDH activity (Figs. 2G and 3). Cell-cycle analysis showed that many YPK-Lm preferentially stayed in the G0/G1 phase (Fig. 4). mRNA levels of mesenchymal markers such as SNAI1 and ZEB2 were expressed in YPK-Lm as we expected (Fig. 5C). Similarly, RT-PCR resulted in higher expression levels of stemness marker such as KIT and ALDH1A1 from YPK-Lm (Fig. 5A). These results suggest that YPK-Lm acquired stemness properties through the EMT. The expression level of $\mathrm{CDH} 1$, which is an epithelium-related gene expected to be high in YPK based on this EMT theory, was high in YPK-Lm (Fig. 5B). Thus, this theory of CSC induction by passage through the EMT still has room for argument. Based on the facts described above, we confirmed that these induced cells have CSCs characteristics. 
A prominent feature of CSCs is their ability to form floating spheroids in serum-free culture conditions (26). Several studies have suggested that CSCs can be enriched in spheres when cultured in serum-free medium supplemented with adequate mitogens, such as bFGF and EGF (27-30). However, culture cells kept in the sphere formation for $>10$ days forfeit not only their stemness properties but also viability. The most problematic issue is the spontaneous differentiation and cell death that accompany stem cell divisions in the sphere environment (31). In contrast, most individual cells in adherent culture conditions are uniformly exposed to defined growth factors and oxygen tension, which allows most CSCs to maintain their stemness properties without spontaneous differentiation and cell death. The laminins are an important and biologically active part of the basal lamina, influencing cell differentiation, migration, and adhesion, as well as survival $(32,33)$. To overcome the limitations of the neurosphere culture paradigm, Pollard et al cultured glioma tumor-initiating cells as adherent cell lines by using laminin-coated dishes (31). In our experiment, the modified stem cell medium with NSF-1, and LIF induced a P-CSLC-enriched population, however, the medium without NSF-1 and/or LIF failed to induce this population (Fig. 2I, and $\mathbf{J}$ ). In addition, this induced population did not divide and the number of cells did not increase in this condition without transferring to laminin-coated dishes. This population has to be transferred to laminin-coated dishes approximately one week after sphere formation. Then, this population is able to maintain the stemness properties and viability with selfrenewing properties. We suggest that the process of CSLC induction demands the neural stimulation factors with some adequate cytokines and chemokines, such as bFGF and EGF. Based on our data of cytokines from the supernatant, it was established that induced and maintained conditions between CSCs and cancer cells are drastically different in terms of cytokines profile in the culture (Fig. 6). As typical examples, b-FGF, IL-9, IP-10, RANTES, and G-CSF were higher in supernatant of CSCs culturing, while TGF- $\beta 1$, TGF- $\beta 3$, IL-5, IL-12, and PDGF-BB were higher in supernatant of cancer cells culturing. Needless to say, this part of the study is immature and weak. Further analysis and study will be required to reveal the mechanism inducing CSLCs in the culture.

Currently, CSC-targeting therapy has been attempted to be established $(34,35)$, because conventional anticancer treatments do not target CSCs and have no efficacy against CSCs. However, one of the difficulties in the quest to characterize the CSC population from tumor specimens is the rarity of this population. Using the method as established in this study, we can easily enrich the CSLC population without special instruments. Although this method is potentially able to be applied to freshly harvested cancer tissue, further investigations in this area are needed. We are planning to use these induced cells to establish a novel immunotherapy targeting CSCs through proteomics. For screening the ability of the immune effector cells to eradicate their target-CSCs, an appropriate number of CSCs can be used with this novel technology.

In conclusion, we established a culture method to induce a CSLC-enriched population from human pancreatic cancer cell lines. This method may be useful to analyze CSC characteristics in detail, and to help in the establishment of novel therapies against CSCs.

\section{Acknowledgements}

We thank Hirokazu Sadahiro and Moeko Inoue for technical support. This study was supported by Japan Society for the Promotion of Science KAKENHI grants 24390317 (to M.O.) and Yamaguchi University research grant Project of Priming Water (Strategic Research Promotion Project) (to K.Y.) .

\section{References}

1. Reya T, Morrison SJ, Clarke MF and Weissman IL: Stem cells, cancer, and cancer stem cells. Nature 414: 105-111, 2001.

2. Visvader JE and Lindeman GJ: Cancer stem cells in solid tumours: accumulating evidence and unresolved questions. Nat Rev Cancer 8: 755-768, 2008.

3. Costello RT, Mallet F, Gaugler B, et al: Human acute myeloid leukemia $\mathrm{CD} 34^{+} / \mathrm{CD} 38^{-}$progenitor cells have decreased sensitivity to chemotherapy and Fas-induced apoptosis, reduced immunogenicity, and impaired dendritic cell transformation capacities. Cancer Res 60: 4403-4411, 2000.

4. Dean M, Fojo T and Bates S: Tumour stem cells and drug resistance. Nat Rev Cancer 5: 275-284, 2005.

5. Guzman ML, Swiderski CF, Howard DS, et al: Preferential induction of apoptosis for primary human leukemic stem cells. Proc Natl Acad Sci USA 99: 16220-16225, 2002.

6. Siegel R, Naishadham D and Jemal A: Cancer statistics, 2012. CA Cancer J Clin 62: 10-29, 2012.

7. Li C, Heidt DG, Dalerba P, et al: Identification of pancreatic cancer stem cells. Cancer Res 67: 1030-1037, 2007.

8. Hermann PC, Huber SL, Herrler T, et al: Distinct populations of cancer stem cells determine tumor growth and metastatic activity in human pancreatic cancer. Cell Stem Cell 1: 313-323, 2007.

9. Eramo A, Lotti F, Sette G, et al: Identification and expansion of the tumorigenic lung cancer stem cell population. Cell Death Differ 15: 504-514, 2008.

10. Kondo T, Setoguchi T and Taga T: Persistence of a small subpopulation of cancer stem-like cells in the C6 glioma cell line. Proc Natl Acad Sci USA 101: 781-786, 2004.

11. Wu C and Alman BA: Side population cells in human cancers. Cancer Lett 268: 1-9, 2008.

12. Ricci-Vitiani L, Lombardi DG, Pilozzi E, et al: Identification and expansion of human colon-cancer-initiating cells. Nature 445: 111-115, 2007.

13. Dontu G, Abdallah WM, Foley JM, et al: In vitro propagation and transcriptional profiling of human mammary stem/progenitor cells. Genes Dev 17: 1253-1270, 2003.

14. Yamamoto K, Yahara N, Gondo T, Ishihara T and Oka M: Establishment and characterization of a new human pancreatic cancer cell line, YPK-1. Bull Yamaguchi Med Sch 49: 33-42, 2002.

15. Tsunedomi R, Iizuka N, Tamesa T, et al: Decreased ID2 promotes metastatic potentials of hepatocellular carcinoma by altering secretion of vascular endothelial growth factor. Clin Cancer Res 14: 1025-1031, 2008

16. Tsunedomi R, Iizuka N, Harada S and Oka M: Susceptibility of hepatoma-derived cells to histone deacetylase inhibitors is associated with ID2 expression. Int J Oncol 42: 1159-1166, 2013.

17. Nagano O, Okazaki S and Saya H: Redox regulation in stem-like cancer cells by CD44 variant isoforms. Oncogene 32: 5191-5198, 2013.

18. Tanabe KK, Ellis LM and Saya H: Expression of CD44R1 adhesion molecule in colon carcinomas and metastases. Lancet 341: 725-726, 1993.

19. Abdullah LN and Chow EK: Mechanisms of chemoresistance in cancer stem cells. Clin Transl Med 2: 3, 2013.

20. Dick JE: Stem cell concepts renew cancer research. Blood 112: 4793-4807, 2008.

21. Thiery JP, Acloque H, Huang RY and Nieto MA: Epithelialmesenchymal transitions in development and disease. Cell 139: 871-890, 2009.

22. Prat A, Parker JS, Karginova O, et al: Phenotypic and molecular characterization of the claudin-low intrinsic subtype of breast cancer. Breast Cancer Res 12: R68, 2010.

23. Polyak K and Weinberg RA: Transitions between epithelial and mesenchymal states: acquisition of malignant and stem cell traits. Nat Rev Cancer 9: 265-273, 2009. 
24. Mani SA, Guo W, Liao MJ, et al: The epithelial-mesenchymal transition generates cells with properties of stem cells. Cell 133: 704-715, 2008

25. Morel AP, Lievre M, Thomas C, Hinkal G, Ansieau S and Puisieux A: Generation of breast cancer stem cells through epithelial-mesenchymal transition. PLoS One 3: e2888, 2008.

26. Mor G, Yin G, Chefetz I, Yang Y and Alvero A: Ovarian cancer stem cells and inflammation. Cancer Biol Ther 11: 708-713, 2011.

27. Chiou SH, Yu CC, Huang CY, et al: Positive correlations of Oct-4 and Nanog in oral cancer stem-like cells and high-grade oral squamous cell carcinoma. Clin Cancer Res 14: 4085-4095, 2008.

28. Lee J, Kotliarova S, Kotliarov Y, et al: Tumor stem cells derived from glioblastomas cultured in bFGF and EGF more closely mirror the phenotype and genotype of primary tumors than do serum-cultured cell lines. Cancer Cell 9: 391-403, 2006.

29. Hueng DY, Sytwu HK, Huang SM, Chang C and Ma HI: Isolation and characterization of tumor stem-like cells from human meningiomas. J Neurooncol 104: 45-53, 2011.
30. Zhong Y, Guan K, Guo S, et al: Spheres derived from the human SK-RC-42 renal cell carcinoma cell line are enriched in cancer stem cells. Cancer Lett 299: 150-160, 2010.

31. Pollard SM, Yoshikawa K, Clarke ID, et al: Glioma stem cell lines expanded in adherent culture have tumor-specific phenotypes and are suitable for chemical and genetic screens. Cell Stem Cell 4: 568-580, 2009.

32. Ziober AF, Falls EM and Ziober BL: The extracellular matrix in oral squamous cell carcinoma: friend or foe? Head Neck 28: 740-749, 2006.

33. Timpl R, Rohde H, Robey PG, Rennard SI, Foidart JM and Martin GR: Laminin - a glycoprotein from basement membranes. J Biol Chem 254: 9933-9937, 1979.

34. Calabrese C, Poppleton H, Kocak M, et al: A perivascular niche for brain tumor stem cells. Cancer Cell 11: 69-82, 2007.

35. Takeishi S, Matsumoto A, Onoyama I, Naka K, Hirao A and Nakayama KI: Ablation of Fbxw7 eliminates leukemia-initiating cells by preventing quiescence. Cancer Cell 23: 347-361, 2013. 